\title{
Purity and Uncertainty Study of CRM Betulin by DSC
}

\author{
$\mathrm{Li}_{\text {Zhang }}{ }^{1}$ (1) $\cdot$ Peng-Hui Yuan ${ }^{1} \cdot$ De-Zhi Yang ${ }^{1} \cdot$ Yan-Cai Bi ${ }^{2} \cdot$ Bin Su$^{2} \cdot$ Bao-Xi Zhang ${ }^{1} \cdot$ Fu-Qing Wang ${ }^{3} \cdot$ Yang Lu $^{1}$. \\ Guan-Hua Du ${ }^{4}$
}

Received: 2 July 2020 / Accepted: 1 August 2020 / Published online: 18 August 2020

(c) The Author(s) 2020

\begin{abstract}
Betulin (BE) can be obtained from many plants, such as those belonging Betulaceae family, and pharmacological investigations showed its notable biological properties and good potential for food and pharmaceutical development. We investigated the homogeneity, stability, purity, and uncertainty of a newly certified reference material (CRM) of BE. The certified purity value for the CRM of $\mathrm{BE}$ was $99.56 \%$ with an extended uncertainty of $0.07 \%(\mathrm{k}=2, \mathrm{P}=0.95)$, as determined by differential scanning calorimetry (DSC). In this study, DSC was used for the first time for purity determination of BE. Given its high accuracy, precision, and reproducibility, DSC can be used as an alternative technique for purity determination of CRMs in the pharmaceutical and food industry.
\end{abstract}

\section{Graphic abstract}

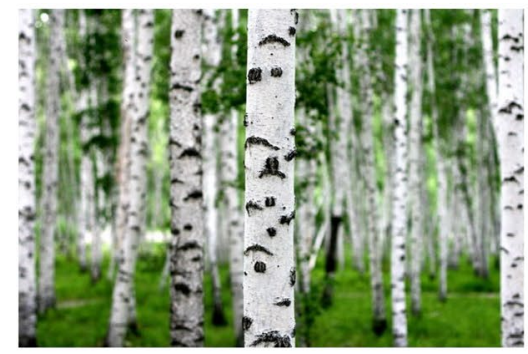

the bark of birch trees

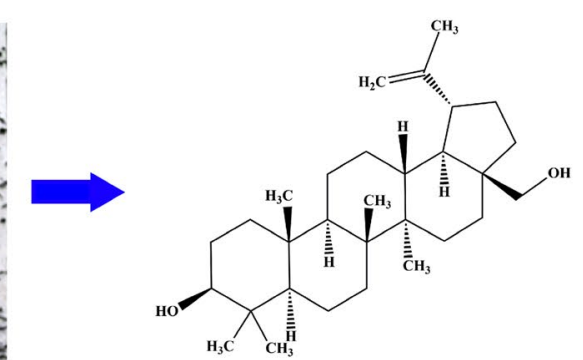

the structural formula of Betulin

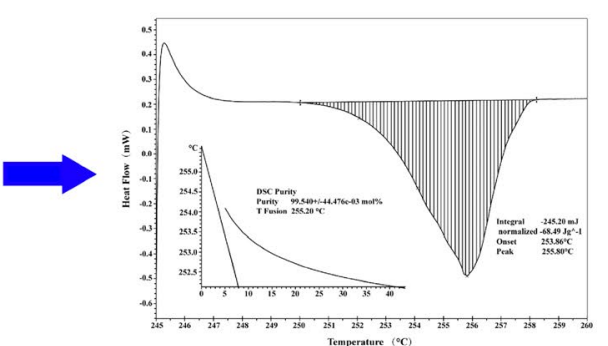

the purity determination curve by DSC of Betulin

Keywords Betulin - DSC · Purity determination - Uncertainty study

Fu-Qing Wang

wangfuqingwfq@163.com

1 Beijing City Key Laboratory of Polymorphic Drugs, Center of Pharmaceutical Polymorphs, Institute of Materia Medica, Chinese Academy of Medical Sciences and Peking Union Medical College, Beijing 100050, People's Republic of China

2 Soteria Pharmaceutical Co., Ltd., Laiwu 271100, People's Republic of China

3 China Pharmaceutical Enterprises Association, Beijing 100044, People's Republic of China

4 Beijing City Key Laboratory of Drug Target and Screening Research, National Center for Pharmaceutical Screening, Institute of Materia Medica, Chinese Academy of Medical Sciences and Peking Union Medical College, Beijing 100050, People's Republic of China

\section{Introduction}

Betulin [BE; betulinol, 3ß-lup-20(29)-en-3,28-diol] belongs to a class of pentacyclic triterpenes and is the predominant compound of the outer bark of birch tree (Betula) [1]. Figure 1 shows the structural formula of BE. BE can be obtained from more than 200 plants species, although the richest source of $\mathrm{BE}$ are those belonging to Betulaceae family, especially Betula alba, B. pubescens, B. platyphylla, and $B$. pendula [2]. Pharmacological investigations have revealed that $\mathrm{BE}$ has many notable biological properties, such as anti-bacterial, anti-inflammatory, anti-fungal, and anti-viral effects, that work effectively on lesions; it also 


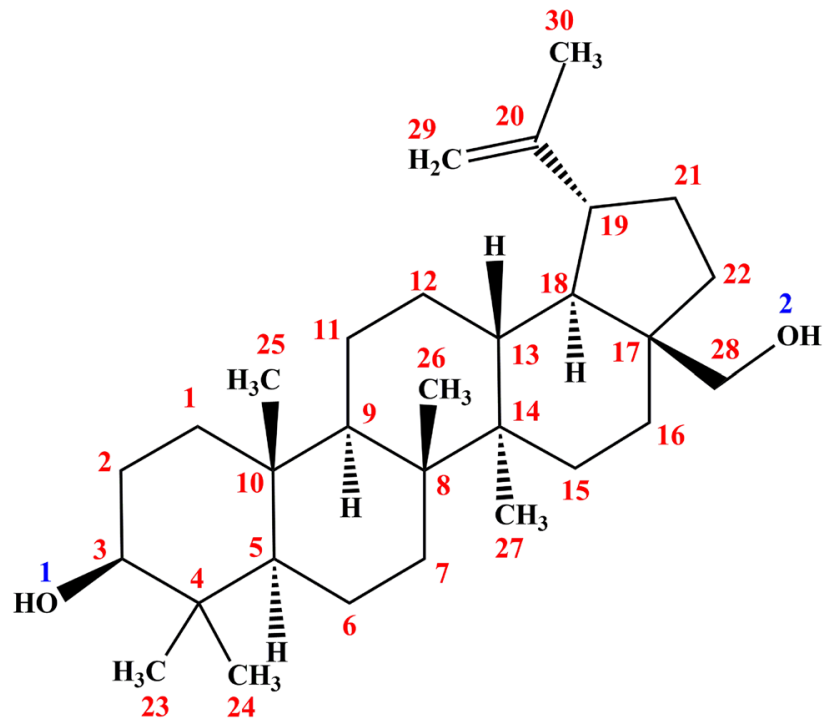

Fig. 1 Structural formula of Betulin

acts as a prophylactic and curative agent [3]. BE exhibits antitumor activity against many types of tumor cell lines: cervix carcinoma HeLa cells, hepatoma HepG2 cells, lung adenocarcinoma A549 cells, breast cancer MCF-7 cells, human gastric carcinoma, human pancreatic carcinoma, and different melanoma and skin cancer cell lines $[4,5]$. As a promising clinical medication, $\mathrm{BE}$ has received increasing attention over the past few decades. Hence, the establishment of an accurate quantitative analysis method for BE is essential to enhance its utilization.

Certified reference materials (CRMs) are used for qualitative and quantitative analyses and have extremely important applications in the food and pharmaceutical fields [6]. In addition to accuracy and precision, which are the classical aims of analytical chemistry, resolving uncertainty is a recent and modern goal of CRMs [7, 8]. Therefore, not only the purity of a CRM but also its uncertainty value must be known. The concept of uncertainty can reveal the quantitative interplay of the individual working steps of a method and thus lead to a deep understanding of its critical points.

Traditionally, chromatographic techniques, such as highperformance liquid chromatography (HPLC), in conjunction with other analytical techniques have been used to determine the purity of CRMs. The purity of BE can be determined by HPLC [9-12]. Recently, differential scanning calorimetry (DSC) has been used to quantitatively determine the purity of compounds that are at least 98\% [13].

This paper describes the development process of the $\mathrm{CRM}$ of $\mathrm{BE}$, including the preparation process of the material, evaluation of the homogeneity and stability of samples within and between groups, and employment of a strategy to assign reference values for the CRM. Following ISO Guide 34 and $35[14,15]$ the procedures included qualitative and
Table 1 Measurement data from the investigation of sample weighing of Betulin

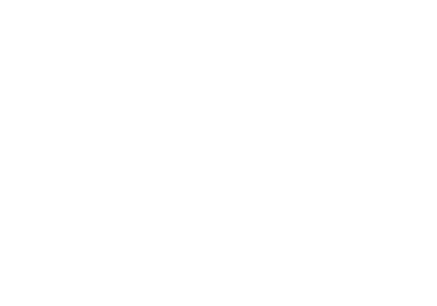

\begin{tabular}{lll}
\hline No. & $\begin{array}{l}\text { Sample } \\
\text { weight } \\
(\mathrm{mg})\end{array}$ & $\begin{array}{l}\text { Endothermic } \\
\text { peak area }(\mathrm{Mj})\end{array}$ \\
\hline 1 & 1.97 & 138.98 \\
2 & 2.21 & 154.68 \\
3 & 2.81 & 198.36 \\
4 & 3.27 & 225.11 \\
5 & 3.68 & 251.13 \\
6 & 4.12 & 281.93 \\
\hline
\end{tabular}

quantitative analyses, verification, the study of homogeneity and stability, purity determination, and uncertainty evaluation. Additionally, in the current article, the CRM of BE was investigated and described by DSC for the first time.

\section{Results and Discussion}

\subsection{Investigation of Heating Rate}

The present study investigated three different heating rates $(0.3,0.5$, and $0.7 \mathrm{~K} / \mathrm{min})$ for purity measurement. The purity values under three different heating rates were all $99.55 \%$. Results showed that the purity value during the measurements was stable and had good parallelism when the heating rate was between $0.3-0.7 \mathrm{~K} / \mathrm{min}$. Therefore, the final experimental conditions were determined at heating rate of $0.5 \mathrm{~K} / \mathrm{min}$ and heating interval of $245-260^{\circ} \mathrm{C}$. Table 1 lists the measurement data from the investigation of heating rate.

\subsection{Investigation of Sample Weighing}

A standard curve was drawn by using the sample mass as the $\mathrm{x}$-coordinate and the endothermic peak area as the y-coordinate. Within the mass range of $1.97-4.12 \mathrm{mg}$, the regression linear equation was $\mathrm{Y}=66.0284 \mathrm{X}+9.6196$, and the correlation coefficient $R^{2}$ was $0.9991(\mathrm{n}=6)$. The results indicated a good linear relationship between the sample mass and endothermic peak area. Consequently, DSC can be used for determining the purity of the CRM of BE. Figure 2 shows the linear regression result of the investigation of sample weighing.

\subsection{Homogeneity Test}

The means of the squares between groups $\left(\mathrm{MS}_{\text {between }}\right)$ and within each group ( $\mathrm{MS}_{\text {within }}$ ) were $6.99 \times 10^{-9}$ and $4.47 \times 10^{-9}$, respectively. The calculated F-value was 1.46 , which was lower than the critical F-value (1.74). No significant differences were found in the purity of BE during the 


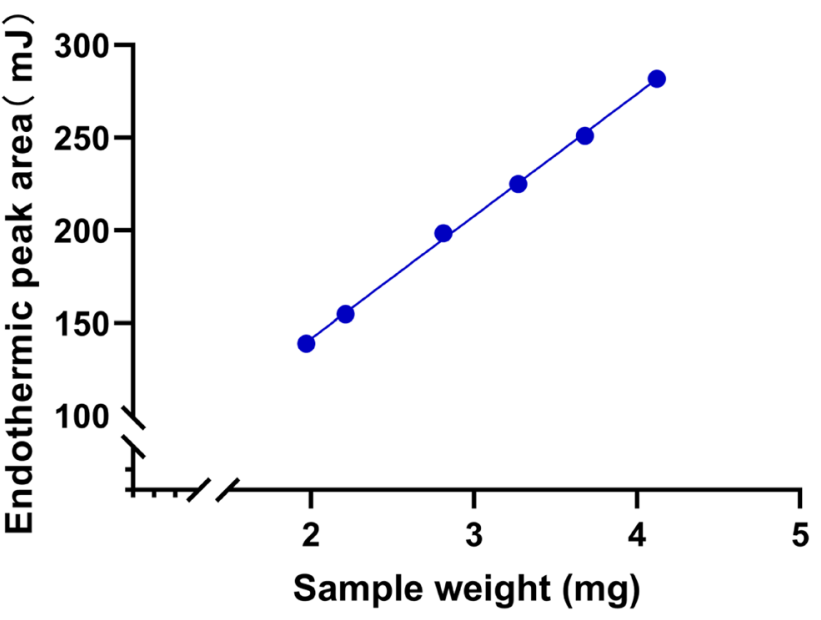

Fig. 2 Linear regression result of the investigation of sample weighing of Betulin

Table 2 Measurement data from the homogeneity test of Betulin

\begin{tabular}{|c|c|c|c|c|}
\hline \multirow[t]{2}{*}{ No. } & \multicolumn{3}{|c|}{ Relative purity $\left(Y_{i j}\right)(\%)$} & \multirow[t]{2}{*}{$\bar{Y}_{j}(\%)$} \\
\hline & Samp1 & Samp2 & Samp3 & \\
\hline 1 & 99.54 & 99.54 & 99.55 & 99.54 \\
\hline 2 & 99.53 & 99.54 & 99.54 & 99.53 \\
\hline 3 & 99.54 & 99.55 & 99.55 & 99.54 \\
\hline 4 & 99.53 & 99.54 & 99.53 & 99.53 \\
\hline 5 & 99.54 & 99.55 & 99.55 & 99.54 \\
\hline 6 & 99.53 & 99.54 & 99.55 & 99.54 \\
\hline 7 & 99.54 & 99.53 & 99.53 & 99.53 \\
\hline 8 & 99.54 & 99.54 & 99.53 & 99.53 \\
\hline 9 & 99.53 & 99.53 & 99.54 & 99.53 \\
\hline 10 & 99.54 & 99.54 & 99.55 & 99.54 \\
\hline 11 & 99.55 & 99.55 & 99.54 & 99.54 \\
\hline 12 & 99.53 & 99.55 & 99.54 & 99.54 \\
\hline 13 & 99.54 & 99.53 & 99.53 & 99.53 \\
\hline 14 & 99.55 & 99.54 & 99.54 & 99.54 \\
\hline 15 & 99.54 & 99.54 & 99.55 & 99.54 \\
\hline 16 & 99.53 & 99.54 & 99.55 & 99.54 \\
\hline 17 & 99.54 & 99.54 & 99.54 & 99.54 \\
\hline 18 & 99.55 & 99.53 & 99.52 & 99.53 \\
\hline 19 & 99.54 & 99.55 & 99.54 & 99.54 \\
\hline 20 & 99.54 & 99.54 & 99.55 & 99.54 \\
\hline 21 & 99.53 & 99.53 & 99.54 & 99.53 \\
\hline 22 & 99.53 & 99.54 & 99.53 & 99.53 \\
\hline 23 & 99.54 & 99.54 & 99.55 & 99.54 \\
\hline 24 & 99.53 & 99.54 & 99.54 & 99.53 \\
\hline 25 & 99.54 & 99.55 & 99.54 & 99.54 \\
\hline Average & & & & 99.54 \\
\hline SD & & & & $4.83 \times 10^{-5}$ \\
\hline
\end{tabular}

homogeneity test. Table 2 provides the measurement data from the homogeneity tests.

The analytical data were assessed by one-way analysis of variance.

\subsection{Stability Test}

The data for the short-term stability tests were examined by Student's t-test, and those for the long-term stability test were assessed by regression analysis. The statistical analysis showed that the CRM of BE can be stored at $25^{\circ} \mathrm{C}$ for at least 12 months. Table 3 lists the short-term stability test measurement data. Figure 3 exhibits the results of long-term stability test of BE.

\subsection{Minimum Sampling Weight Test}

In this part, DSC was used to investigate the minimum sampling weight of the CRM of BE. In the experiment, four different weights, namely, 1.0, 2.0, 3.0, and $4.0 \mathrm{mg}$, were used to study the minimum sampling weight. Six parallel experiments were conducted for each mass, and the results are shown in Table 4.

The results by calculations were as follows: $t_{10}^{0.05}(1.0$, $2.0)=3.475, t_{10}^{0.05}(2.0,3.0)=1.803, t_{10}^{0.05}(2.0,4.0)=1.698$, and $t_{10}^{0.05}(3.0,4.0)=0.360$. The results of t-test showed that the minimum sample quantity of "betulin purity standard material" was $2.0 \mathrm{mg}$.

\subsection{Purity Determination}

Figure 4 illustrates the DSC curve of BE and the endothermic melting peak at $256.5^{\circ} \mathrm{C}$. The purity determination by DSC was based on Van't Hoff's law [16]. The purity of BE was calculated in accordance with the following formula:

$x_{D S C}=\left(1-\frac{\left(T_{0}-T_{m}\right) \Delta H}{R T_{0}^{2}}\right) \times 100 \%=\left(1-\frac{Q M F\left(T_{0}-T_{m}\right)}{m R T_{0}^{2}}\right) \times 100 \%$

where $x_{\mathrm{DSC}}$ is the purity, and $Q$ and $m$ are the thermal values calibrated by stannum and sample mass, respectively. $M$ is the molar mass of $\mathrm{BE} . \mathrm{T}_{0}$ is the melting temperature of the pure compound; $\mathrm{T}_{\mathrm{m}}$ is the actual melting temperature; $\mathrm{R}$ is gas constant; $\Delta \mathrm{H}$ is the molar enthalpy of melting, and $\mathrm{y}$ is the mole fraction of impurity in the substance.

\subsection{Value Characterization}

The purity of the analyte was calculated, and the results of purity determination are listed in Table 5 . The mean purity was $99.56 \%$ with a standard deviation of $1.906 \%$. 
Table 3 Measurement data from the short-term stability test of Betulin

\begin{tabular}{|c|c|c|c|c|c|c|c|c|c|c|c|c|}
\hline \multirow[t]{3}{*}{ Condition } & \multirow[t]{3}{*}{ Time (day) } & \multicolumn{9}{|c|}{ Purity (\%) } & \multirow[t]{3}{*}{ Mean $(\%)$} & \multirow[t]{3}{*}{ SD } \\
\hline & & \multicolumn{3}{|c|}{ Vials 1} & \multicolumn{3}{|c|}{ Vials 2} & \multicolumn{3}{|c|}{ Vials 3} & & \\
\hline & & 1 & 2 & 3 & 1 & 2 & 3 & 1 & 2 & 3 & & \\
\hline \multirow[t]{5}{*}{$60{ }^{\circ} \mathrm{C}$} & 0 & 99.53 & 99.54 & 99.55 & 99.54 & 99.55 & 99.54 & 99.53 & 99.54 & 99.53 & 99.54 & 0.000078 \\
\hline & 5 & 99.54 & 99.53 & 99.54 & 99.53 & 99.54 & 99.55 & 99.54 & 99.55 & 99.55 & 99.54 & 0.000078 \\
\hline & 10 & 99.55 & 99.55 & 99.56 & 99.53 & 99.56 & 99.56 & 99.55 & 99.53 & 99.54 & 99.55 & 0.000120 \\
\hline & 15 & 99.55 & 99.53 & 99.54 & 99.54 & 99.55 & 99.54 & 99.54 & 99.53 & 99.53 & 99.54 & 0.000078 \\
\hline & 20 & 99.54 & 99.55 & 99.55 & 99.54 & 99.55 & 99.54 & 99.53 & 99.54 & 99.56 & 99.54 & 0.000088 \\
\hline \multirow{5}{*}{$\begin{array}{l}\text { Strong light } \\
(45001 \times \pm 5001 \times)\end{array}$} & 0 & 99.54 & 99.55 & 99.53 & 99.55 & 99.54 & 99.55 & 99.54 & 99.55 & 99.55 & 99.54 & 0.000073 \\
\hline & 5 & 99.55 & 99.54 & 99.54 & 99.53 & 99.54 & 99.55 & 99.55 & 99.54 & 99.56 & 99.54 & 0.000088 \\
\hline & 10 & 99.55 & 99.53 & 99.55 & 99.53 & 99.53 & 99.55 & 99.55 & 99.54 & 99.55 & 99.54 & 0.000097 \\
\hline & 15 & 99.54 & 99.54 & 99.54 & 99.55 & 99.56 & 99.54 & 99.56 & 99.56 & 99.54 & 99.55 & 0.000097 \\
\hline & 20 & 99.55 & 99.54 & 99.55 & 99.54 & 99.54 & 99.55 & 99.54 & 99.54 & 99.55 & 99.54 & 0.000053 \\
\hline
\end{tabular}

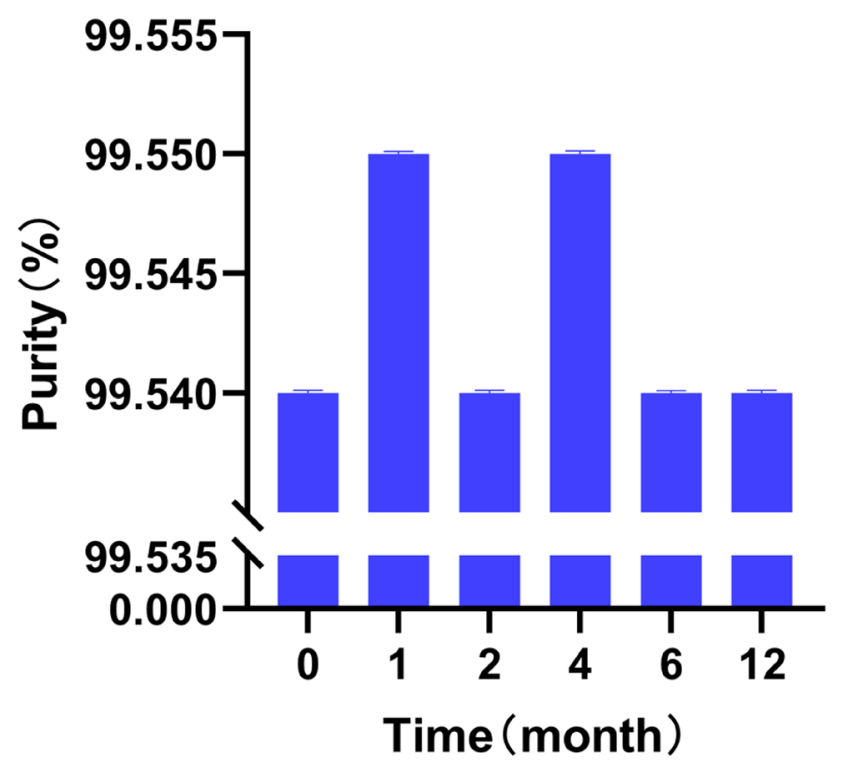

Fig. 3 Results of long-term stability test of Betulin

\subsection{Uncertainty Evaluation}

The factors that affect the accuracy of the results in a measurement include environmental conditions, operational conditions, accuracy in weighing, the instrument used for measurement, molar melting enthalpy analysis, and accuracy of the analytical software. The analysis indicated that the uncertainty of measurement was derived from the following factors: the uncertainty in measurements of the melting enthalpy of stannum, measurement error, weighing error, temperature error, and calculation error of the DSC analysis software.
Table 4 Result of the minimum sampling amount test

\begin{tabular}{|c|c|c|c|}
\hline No. & Weight (mg) & Purity (\%) & No. \\
\hline 0.94 & 99.09 & 99.18 & 0.0026857 \\
\hline 1.09 & 98.79 & & \\
\hline 1.06 & 99.29 & & \\
\hline 1.10 & 99.61 & & \\
\hline 1.16 & 99.11 & & \\
\hline 1.10 & 99.19 & & \\
\hline 2.30 & 99.56 & 99.56 & 0.0001163 \\
\hline 1.91 & 99.55 & & \\
\hline 2.06 & 99.56 & & \\
\hline 2.12 & 99.55 & & \\
\hline 1.96 & 99.58 & & \\
\hline 1.96 & 99.57 & & \\
\hline 2.96 & 99.53 & 99.55 & 0.0001001 \\
\hline 3.02 & 99.55 & & \\
\hline 3.17 & 99.55 & & \\
\hline 3.08 & 99.56 & & \\
\hline 3.00 & 99.56 & & \\
\hline 3.14 & 99.55 & & \\
\hline 4.02 & 99.56 & 99.55 & 0.0000775 \\
\hline 3.90 & 99.55 & & \\
\hline 3.99 & 99.55 & & \\
\hline 3.88 & 99.56 & & \\
\hline 3.98 & 99.54 & & \\
\hline 3.91 & 99.55 & & \\
\hline
\end{tabular}

\subsubsection{Uncertainty from Melting Enthalpy Measurements of Stannum $\left(\mathrm{u}_{\mathrm{B} 1(\mathrm{DSC})}\right)$}

Table 6 lists the melting enthalpy of stannum $\left(u_{\mathrm{B} 1(\mathrm{DSC})}\right)$ measured by DSC. 


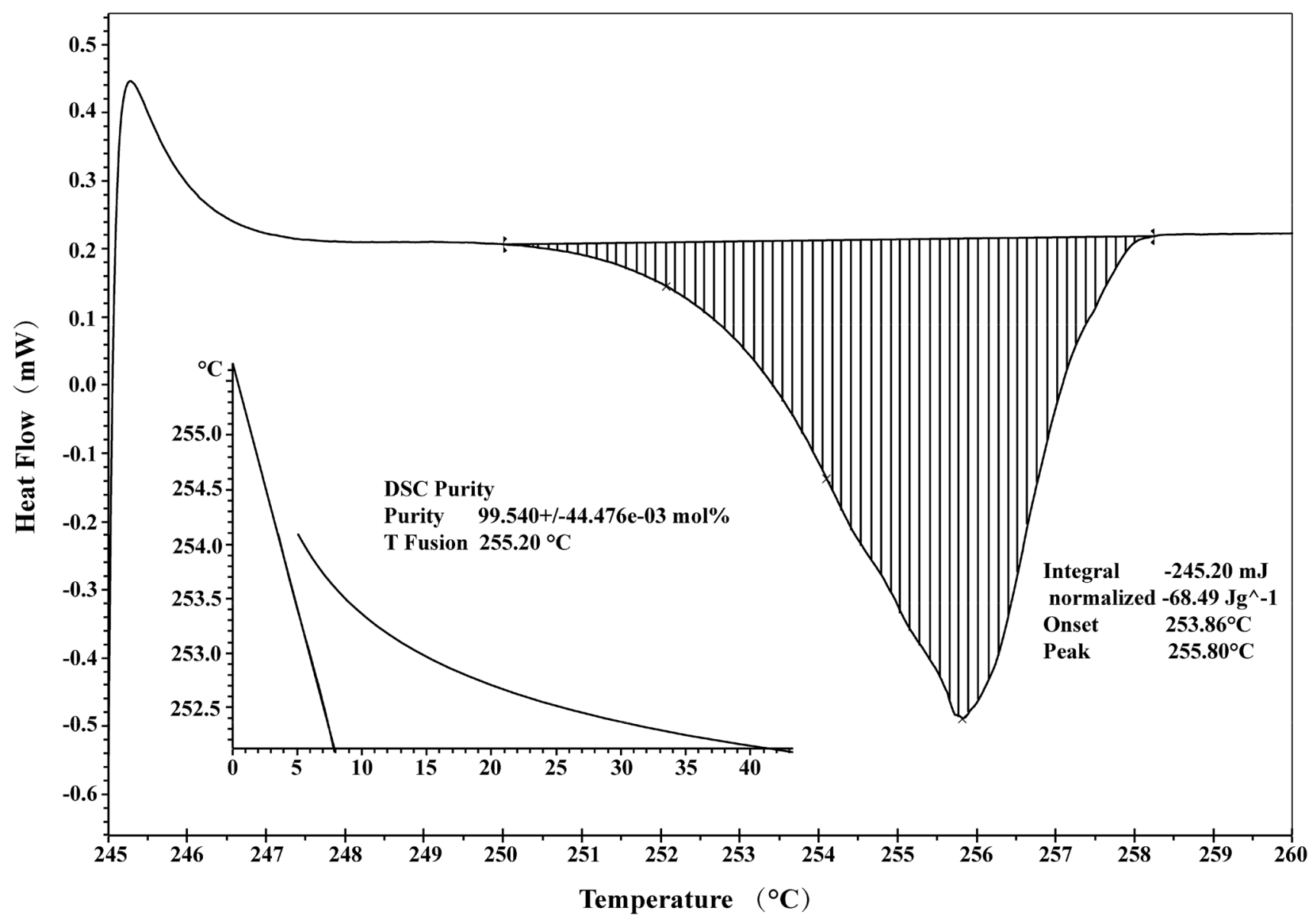

Fig. 4 The DSC curve of Betulin

Table 5 Purity of analyte as determined by the DSC method

\begin{tabular}{lll}
\hline No. & Weight $(\mathrm{mg})$ & Purity (\%) \\
\hline 1 & 3.58 & 99.54 \\
2 & 3.54 & 99.55 \\
3 & 3.35 & 99.57 \\
4 & 3.25 & 99.59 \\
5 & 3.43 & 99.56 \\
6 & 3.34 & 99.56 \\
7 & 3.48 & 99.54 \\
8 & 3.54 & 99.58 \\
9 & 3.33 & 99.53 \\
10 & 3.59 & 99.55 \\
\hline
\end{tabular}

Table 6 Melting enthalpy of Stannum measured by the DSC method

\begin{tabular}{lll}
\hline No. & $\begin{array}{l}\text { Melting } \\
\text { enthalpy } \\
(\mathrm{J} / \mathrm{g})\end{array}$ & SD \\
\hline 1 & -59.70 & 0.0004448 \\
2 & -59.70 & 0.0004127 \\
3 & -59.70 & 0.0004184 \\
4 & -59.69 & 0.0003722 \\
5 & -59.72 & 0.0004161 \\
6 & -59.69 & 0.0004044 \\
7 & -59.70 & 0.0004250 \\
8 & -59.70 & 0.0003955 \\
9 & -59.70 & 0.0004078 \\
10 & -59.71 & 0.0003906
\end{tabular}

The uncertainty of $u_{\mathrm{B} 1 \text { (DSC) }}$ was calculated using the following expression: 
$u_{B 1(D S C)}=\frac{s}{\sqrt{n} \times \overline{\Delta H}}=4.64 \times 10^{-5}$.

\subsubsection{Uncertainty from Measurement Error $\left(\mathrm{u}_{\mathrm{A} 1(\mathrm{DSC})}\right)$}

The $u_{\text {Al(DSC) }}$ was calculated using the following expression:

$u_{\mathrm{A} 1(\mathrm{DSC})}=\frac{s}{\sqrt{n} \times \bar{P}}=6.03 \times 10^{-5}$

\subsubsection{Uncertainty from Weighing Error ( $\left.u_{\text {B2(DSC) }}\right)$}

The $u_{\mathrm{B} 2(\mathrm{DSC})}$ was calculated using the following expression:

$u_{B 2(D S C)}=\frac{\left(\frac{\partial X}{\partial m} d m\right)}{k \times m_{\min }}=-8.575 \times 10^{-5}$

\subsubsection{Uncertainty from Temperature Error ( $\left.\mathrm{u}_{\mathrm{B} 3(\mathrm{DSC})}\right)$}

The $u_{\mathrm{B} 3(\mathrm{DSC})}$ was calculated using the following expression:

$u_{B 3(D S C)}=\left(\frac{\partial X}{\partial T_{0}}+\frac{\partial X}{\partial T_{m}}\right) / k=1.719 \times 10^{-4}$

\subsubsection{Uncertainty from Systematic Deviation of the DSC Analysis Software $\left(u_{B 4(D S C)}\right)$}

Table 6 shows the systematic deviation of the DSC analysis software from the purity determination.

The $u_{\mathrm{B} 4(\mathrm{DSC})}$ was estimated using the following expression:

$u_{B 4(D S C)}=\frac{d_{\max }}{k \times \bar{P}}=2.579 \times 10^{-4}$.

\subsubsection{Combined Uncertainty of CRM}

The uncertainty of CRM arising from $u_{\mathrm{B} 1 \text { (DSC) }}, u_{\mathrm{Al}(\mathrm{DSC})}, u$ $\mathrm{B} 2(\mathrm{DSC}), u_{\mathrm{B} 3(\mathrm{DSC})}$, and $u_{\mathrm{B} 4(\mathrm{DSC})}$ were calculated and discussed. Figure 5 shows the cause-and-effect diagram revealing the possible sources of uncertainty in DSC.

The combined uncertainty of CRM was calculated using the following expression:

\subsubsection{Expanded Uncertainty (Uc) of CRM}

The $U \mathrm{c}$ of CRM was calculated using a coverage factor of 2 , which is associated with a confidence level of approximately $95 \%$.

$U c(x)=k_{p} u_{(D S C)}=0.07 \%$.

\subsection{Results of CRM Analysis}

The purity of the CRM of BE was estimated at $99.56 \% \pm 0.07 \%(\mathrm{k}=2)$ within a typical $95 \%$ confidence interval based on the combined value assignments and the $U$ c by DSC.

\subsection{Method Validation and Value Certification}

The purity of BE was certified by the mass balance method, which obtained a value of $99.54 \% \pm 0.24 \%(\mathrm{k}=2)$ within a typical $95 \%$ confidence interval. The t-test indicated no significant difference between the results of the two methods. The certified value was expressed as the mean of the purity obtained by the two methods. The certified value of the CRM of BE was $99.6 \% \pm 0.5 \%(\mathrm{k}=2)$ within a typical $95 \%$ confidence interval.

\section{Conclusion}

In this study, a reliable, sensitive, and rapid DSC method was developed for the purity determination of a new CRM of $\mathrm{BE}$. The purity of BE was determined by DSC method and certified by the mass balance method. The results of the two methods were consistent, indicating that DSC method can be used as an alternative technique for the purity determination of materials. The validation data presented here indicate that DSC method can be used to determine the purity of $\mathrm{BE}$ with consistent results. Compared with the traditional mass balance method, DSC method is faster and provides higher accuracy and precision. Furthermore, this method has good reproducibility and requires no corresponding reference standard and sample preparation. DSC method can be used as an alternative reference method to provide accurate data. The results can be compared with those of the routine

$$
\begin{aligned}
\frac{u_{(D S C)}}{P_{(D S C)}} & =\sqrt{u_{B 1(D S C)}^{2}+u_{A 1(D S C)}^{2}+u_{B 2(D S C)}^{2}+u_{B 3(D S C)}^{2}+u_{B 4(D S C)}^{2}}=3.305 \times 10^{-4} \\
u_{(D S C)} & =P_{(D S C)} \times \sqrt{u_{B 1(D S C)}^{2}+u_{A 1(D S C)}^{2}+u_{B 2(D S C)}^{2}+u_{B 3(D S C)}^{2}+u_{B 4(D S C)}^{2}} \\
& =99.56 \% \times 3.305 \times 10^{-4}=3.290 \times 10^{-4}
\end{aligned}
$$




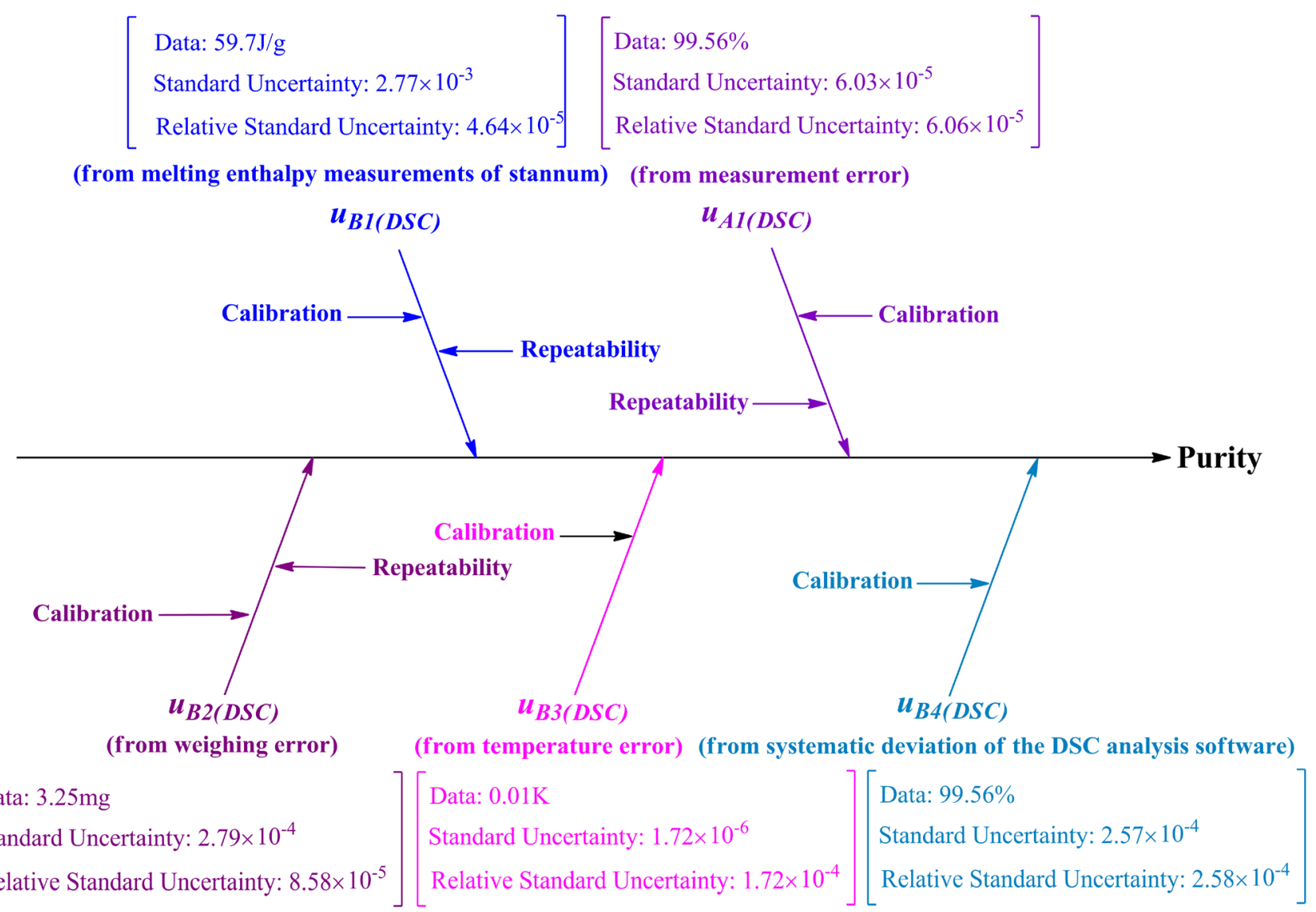

Fig. 5 Cause and effect diagram showing the possible sources of uncertainty in the DSC method

methods employed for testing BE. We envision that DSC method can be used extensively in tests and evaluation processes in the pharmaceutical industry.

The new CRM of BE can be used for the calibration of analytical instruments, assessment of test methods, and securing the accuracy and comparability of measurement data. The new CRM of BE has been approved by the national administrative committee for CRM (GBW09593). This reference material can also be widely used to control the quality of BE-related pharmaceutical and foods formulations.

\section{Experiment Section}

\subsection{Materials}

BE was obtained from Yuancheng Co., Ltd. (Wu Han, China). All other reagents were of analytical grade.

\subsection{Preparation of Reference Material}

BE (50 g) was dissolved in $95 \%$ ethanol at an external temperature of $60{ }^{\circ} \mathrm{C}$ in a round-bottom flask, and then kept at
$60{ }^{\circ} \mathrm{C}$ until the sample had dissolved. Then the solution was subjected to hot filtering and retained at $4{ }^{\circ} \mathrm{C}$ overnight. After the crystals had completely precipitated, they were filtered and vacuum dried to obtain highly pure crystals of $\mathrm{BE}$, which were dried at $60{ }^{\circ} \mathrm{C}$ for $4 \mathrm{~h}$. The powder was milled and then passed through a $74-\mu \mathrm{m}$ sieve. The fraction was homogenized in a rotating mixer prior to filling. Samples of $50 \mathrm{mg}$ of the homogenized powder were dispensed into each dark glass vial, which were then sealed. Six hundred bottles were prepared for each batch of BE. The samples were stored at $25^{\circ} \mathrm{C}$ for studies of homogeneity and stability.

\subsection{Determination of Homogeneity and Stability}

Studies of homogeneity and stability were performed using the DSC method. The between-vial homogeneity was determined by analyzing one replicate in each of the 25 randomly selected bottles from each batch. The within-vial homogeneity was tested in three replicates for each vial. The results were statistically evaluated.

Eighteen samples were selected for the short-term stability study. The samples were divided into six different groups, each group containing three samples. The six groups of the 
sample were respectively stored at $60{ }^{\circ} \mathrm{C}$ and under light conditions $(45001 \times \pm 5001 \times)$ for 0,1 , and 2 weeks. Another 36 samples were selected for the long-term stability study. The samples were divided into six different groups, each group containing six samples. The six groups of the sample were stored at $25^{\circ} \mathrm{C}$ for $0,1,2,4,6$, and 12 months under lighted condition, respectively.

\subsection{Characterization}

The DSC analysis of the samples was conducted using a DSC1 system (Mettler-Toledo Inc, Switzerland). The samples (at approximately 3-5 $\mathrm{mg}$ ) were hermetically sealed in aluminum crucibles to prevent any mass loss in the presence of moisture. All sample measurements were performed in triplicate at a heating rate of $0.5 \mathrm{~K} / \mathrm{min}$ and temperatures ranged from 245 to $260^{\circ} \mathrm{C}$ with furnace gas $\mathrm{N}_{2}$. The purity results obtained by DSC are reported as the average of the triplicate determinations.

Acknowledgements This work was financially supported by National Key R\&D Program of China (Grant No. 2016YFC1000900), National Science and Technology Major Project of China (Grant Nos. 2017ZX09101001003, 2018ZX09711001-003-022, 2018ZX09711001010), National Natural Science Foundation of China(NSFC) (Grant No. 81703473) and CAMS Innovation Fund for Medical Sciences(Grant No. 2017-I2M-3-010).

\section{Compliance with Ethical Standards}

Conflict of interest The Authors declare that there is no conflict of interest.

Open Access This article is licensed under a Creative Commons Attribution 4.0 International License, which permits use, sharing, adaptation, distribution and reproduction in any medium or format, as long as you give appropriate credit to the original author(s) and the source, provide a link to the Creative Commons licence, and indicate if changes were made. The images or other third party material in this article are included in the article's Creative Commons licence, unless indicated otherwise in a credit line to the material. If material is not included in the article's Creative Commons licence and your intended use is not permitted by statutory regulation or exceeds the permitted use, you will need to obtain permission directly from the copyright holder. To view a copy of this licence, visit http://creativecommons.org/licenses/by/4.0/.

\section{References}

1. A. Hordyjewska, A. Ostapiuk, A. Horecka, J. Kurzepa, Phytochem. Rev. 18, 929-951 (2019)

2. C.A. Dehelean, S. Feflea, J. Molnár, I. Zupko, C. Soica, Nat. Prod. Commun. 7, 981-985 (2012)

3. A. Falamas, C.A. Dehelean, S. Cinta pinzaru, Vib. Spectrosc. 95, 44-50 (2018)

4. Z. Hu, Z. Wang, S. Luo, O.J. Margaret, Y. Wang, Chem. Biol. Interact. 302, 190-195 (2019)

5. C. Danciu, I. Pinzaru, D. Coricovac, F. Andrica, I. Sizemore, C. Dehelean, F. Baderca, V. Lazureanu, C. Soica, M. Mioc, H. Radeke, Eur. J. Pharm. Biopharm. 134, 1-19 (2019)

6. D. Yang, L. Jiao, B. Zhang, G. Du, Y. Lu, J. Pharm. Biomed. Anal. 140, 169-173 (2017)

7. R. Borgesa, V.R. Meyer, J. Pharm. Biomed. Anal. 77, 40-43 (2013)

8. E. Rozeta, R.D. Marinia, E. Ziemonsa, B. Boulanger, P. Hubert, J. Pharm. Biomed. Anal. 55, 848-858 (2011)

9. G. Zhao, W. Yan, D. Cao, J. Pharm. Biomed. Anal. 43, 959-962 (2007)

10. A.K. Maji, N. Maity, P. Banerji, D. Banerjee, Int. J. Phytomedicine. 5, 131 (2013)

11. A.K. Ressmann, K. Strassl, P. Gaertner, B. Zhao, L. Greiner, K. Bica, Green Chem. 14, 940-944 (2012)

12. A.K. Maji, S. Pandit, P. Banerji, D. Banerjee, Int. J. Pharm. Pharm. Sci. 6, 691-695 (2014)

13. O. Nara, Anal. Chim. Acta. 685, 91-102 (2011)

14. ISO Guide 34, General requirements for the competence of reference material producers, International Organization for Standardization. (2009)

15. ISO Guide 35, Reference materials-General and statistical principles for certification, International Organization for Standardization. (2006).

16. S. Mathkar, S. Kumar, A. Bystol, K. Olawoore, D. Min, R. Markovich, A. Rustum, J. Pharm. Biomed. Anal. 49, 627-631 (2009) 